\title{
Ultrafast carrier dynamics of epitaxial silicene
}

\author{
Eugenio Cinquanta, ${ }^{1, \dagger}$ Guido Fratesi, ${ }^{2,3}$ Stefano dal Conte, ${ }^{4,5}$ Carlo Grazianetti, ${ }^{1}$ Francesco \\ Scotognella, ${ }^{5, *}$ Salvatore Stagira, ${ }^{5}$ Caterina Vozzi, ${ }^{4}$ Giovanni Onida,${ }^{2,3}$ and Alessandro Molle ${ }^{1, *}$ \\ ${ }^{1}$ Laboratorio MDM, IMM-CNR, via C. Olivetti 2, Agrate Brianza, I-20864, Italy \\ ${ }^{2}$ Dipartimento di Fisica, Universita 'degli Studi di Milano, Via Celoria 16, 20133 Milano, Italy \\ ${ }^{3}$ European Theoretical Spectroscopy Facility, Via Celoria 16, 20133 Milano, Italy \\ ${ }^{4}$ CNR Istituto di Fotonica e Nanoteconologie, Piazza Leonardo da Vinci 32, 20133 Milano, Italy \\ ${ }^{5}$ Dipartimento di Fisica, Politecnico di Milano, Piazza L. da Vinci 32, 20133 Milano, Italy \\ *alessandro.molle@mdm.imm.cnr.it phone+390396032884 fax + 390396881175 \\ *francesco.scotognella@polimi.it; phone +39 022399 6056; fax +39 0223996126 \\ * guido.fratesi@unimi.it; phone+3902 503 17348; fax +3902503 17482 \\ $\dagger$ present address Dipartimento di Fisica, Politecnico di Milano, Piazza L. da Vinci 32, 20133 Milano, Italy
}

\begin{abstract}
The recent integration of silicene in field-effect transistors (FET) opened new challenges in the comprehension of the chemical and physical properties of this elusive two-dimensional allotropic form of silicon. Intense efforts have been devoted to the study of the epitaxial Silicene/ $\operatorname{Ag}(111)$ system in order to elucidate the presence of Dirac fermion in analogy with graphene; strong hybridization effects in silicene superstructures on silver have been invoked as responsible for the disruption of $\pi$ and $\pi^{*}$ bands. In this framework, the measured ambipolar effect in silicene-based FET characterized by a relatively high mobility, points out to a complex physics at the silicene-silver interface, demanding for a deeper comprehension of its details on the atomic scale. Here we elucidate the role of the metallic support in determining the physical properties of the $\mathrm{Si} / \mathrm{Ag}$ interface, by means of optical techniques combined with theoretical calculations of the optical response of the supported system. The silicene/ $\operatorname{Ag}(111)$ spectra, which turn out to be strongly non-additive, are analyzed in the framework of theoretical density functional based calculations allowing us to single out contributions arising from different localization. Electronic transitions involving silver states are found to provide a huge contribution to the optical absorption of silicene on silver, compatible with a strong Si-Ag hybridization. The results point to a dimensionality-driven peculiar dielectric response of the two-dimensional-silicon/silver interface, which is confirmed by means of Transient-Reflectance spectroscopy. The latter shows a metallic-like carrier dynamics, (both for silicene and amorphous silicon), hence providing an optical demonstration of the strong hybridization arising in silicene/Ag(111) systems.
\end{abstract}

Keywords: two-dimensional materials; silicene; ultrafast spectroscopy.

\section{INTRODUCTION}

A very interesting system with "on demand physical properties" is Silicene. Among 2D materials beyond Graphene, Silicene entered the scientific arena as an intriguing option ${ }^{1}$. Free-standing Silicene is theoretically expected to exhibit massless Dirac fermions and tunable electronic properties under a normal electric field ${ }^{1,2}$. Early experiments showed that Silicon atoms arrange on metal surfaces in differently oriented $2 \mathrm{D}$ honeycomb lattices ${ }^{3-5}$. However, electronic band structure (EBS) engineering of silicene is jeopardized by the nontrivial Silicene-substrate interaction resulting from recent Angle-Resolved Photoemission (ARPES) spectroscopy ${ }^{3-7}$. Recently, by combining ab-initio density-functional theory calculation and pump-probe spectroscopy, we demonstrate how the silicene-silver interface is characterized by an intriguing mixing effect affecting silicene and silver electronic wavefunctions ${ }^{8}$. The resulting metallic-like behaviour evidences how epitaxial silicene presents the same drawback of graphene, which is the absence of a band gap. As a consequence, in order to draw a roadmap for the integration of silicene-based optoelectronics devices, the opening of a band gap is mandatory. In this respect, the pioneering integration of Silicene-based Filed-Effect Transistor (FET) showing an ambipolar effect once peeled form the silver substrate ${ }^{9}$, promoted Silicene as a promising platform for the realization of artificial 2D materials with "on demand" physical properties.

Here we elucidate the role of the metallic support in determining the physical properties of the $\mathrm{Si} / \mathrm{Ag}$ interface, by means of optical techniques combined with theoretical calculations of the optical response of the supported system. The

Ultrafast Phenomena and Nanophotonics XXI, edited by Markus Betz, Abdulhakem Y. Elezzabi,

Proc. of SPIE Vol. 10102, 101020J · C 2017 SPIE · CCC code: 0277-786X/17/\$18 doi: $10.1117 / 12.2252009$ 
silicene/ $\operatorname{Ag}(111)$ spectra, which turn out to be strongly non-additive, are analyzed in the framework of theoretical density functional based calculations allowing us to single out contributions arising from different localization. Electronic transitions involving silver states are found to provide a huge contribution to the optical absorption of silicene on silver, compatible with a strong $\mathrm{Si}-\mathrm{Ag}$ hybridization. The results point to a dimensionality-driven peculiar dielectric response of the two-dimensional-silicon/silver interface, which is confirmed by means of Transient-Reflectance spectroscopy. The latter shows a metallic-like carrier dynamics, (both for silicene and amorphous silicon), hence providing an optical demonstration of the strong hybridization arising in silicene/ $\operatorname{Ag}(111)$ systems.

\section{METHODS}

Fabrication and morphological characterization: Samples were grown in ultra-high vacuum system with base pressure in the $10^{10}$ mbar range. The $\mathrm{Ag}(111)$ on mica substrates were cleaned by cycles of $\mathrm{Ar}+$ ion sputtering $\left(1 \mathrm{keV}, 10^{6} \mathrm{mbar}\right)$ and subsequent annealing at $530{ }^{\circ} \mathrm{C}$, until STM showed clean and well-ordered $\mathrm{Ag}$ terraces. Si was deposited by molecular beam epitaxy from a heated crucible with the substrate held at $250{ }^{\circ} \mathrm{C}, 270{ }^{\circ} \mathrm{C}$, and room temperature for the mixed phase (Fig. S1a), $2 \sqrt{3} \times 2 \sqrt{3}$ (Fig. S1b), and amorphous phase (Fig. S1c), respectively. The deposition rate (1.1 $\times 10^{-2} \mathrm{ML} / \mathrm{min}$ ) was estimated by means of a quartz microbalance and by $\mathrm{x}$-ray pho- toelectron spectroscopy. STM topographies were obtained at room temperature using an Omicron STM setup equipped with a chemically etched tungsten tip. Ex-situ measurements were performed on $\mathrm{Al}_{2} \mathrm{O}_{3}$ capped samples. $\mathrm{Al}_{2} \mathrm{O}_{3}$ capping layers were grown by means of $\mathrm{Al}$ deposition in $\mathrm{O}_{2}$ atmosphere as reported in Ref. ${ }^{5}$.

Raman spectroscopy: ex situ Raman characterization by using a Renishaw Invia spectrometer equipped with the 2.5 $\mathrm{eV} / 488 \mathrm{~nm}$ line of an $\mathrm{Ar}^{+}$laser line focused on the sample by a $50 \times 0.75$ N.A. Leica objective providing a spot diameter of about $0.8 \mu \mathrm{m}$. The power at the sample was maintained at $1 \mathrm{~mW}$ in order to prevent laser induced sample heating, and we acquired hundreds of spectra in order to get the highest signal to noise ratio. We carried out all the measurements in a z-backscattering geometry.

Density Functional Theory (DFT) calculations: Optimized coordinates for the adsorbed silicene phases $(4 \times 4$, $\sqrt{13} \times \sqrt{13}$, and $2 \sqrt{3} \times 2 \sqrt{3}$ ) were determined by some of us in a previous paper ${ }^{5}$ and are taken here as a reference. They are depicted in Figs. 2(a), 2(b), and 2(c), respectively. Structural models consist of a five-layer $\operatorname{Ag}(111)$ slab, with silicon atoms adsorbed on one side only. Periodic boundary conditions are imposed, with a vacuum separation between adjacent slabs of at least $17 \AA$. We also considered structural models for amorphous silicon overlayers, using the same computational setup. In this case, the initial structures were obtained by slicing the atomic positions at a cubic$\mathrm{Si} /$ amorphous-Si interface, as determined by tight-binding molecular dynamics simulations ${ }^{10}$, placing it on a $4 \times 4$ $\operatorname{Ag}(111)$ supercell, and performing further geometrical relaxation. This resulted in the structural model shown in Fig. 2(d). The ground state charge density and potential were computed at fixed atomic coordinates by using pseudopotentials and a plane wave basis set, as implemented in the Quantum-ESPRESSO package ${ }^{11}$. We adopt the local density approximation (LDA) to the exchange and correlation functional ${ }^{12}$, with a kinetic energy cutoff of 32 Ry and a $3 \times 3$ surface Brillouin zone sampling. We finally evaluate the optical properties using the Yambo software ${ }^{13}$. We focus on the optical absorbance at normal incidence in the visible-near UV range, which for a silicene $/ \mathrm{Ag}(111)$ slab reads ${ }^{14}$ :

$A(\omega)=\frac{\omega L}{c} \operatorname{Im} \varepsilon_{M}(\omega)$

Here $\mathrm{L}$ is the thickness of the three-dimensional supercell (slab and vacuum space) in the direction orthogonal to the surface, and $\varepsilon_{\mathrm{m}}$ its macroscopic dielectric constant. In the independent particle random phase approximation (IP-RPA) $\varepsilon \mathrm{M}$ is given by a sum of individual valence (v) to conduction (c) transitions involving the velocity operator $\mathrm{v}^{15}$ :

$\operatorname{Im} \varepsilon_{M}(\omega)=\frac{16}{\omega^{2}} \sum_{v, c, k}\left|\left\langle\psi_{v c}|v| \psi_{c k}\right\rangle\right|^{2} \delta\left(\epsilon_{c k}-\epsilon_{v k}-\omega\right)$

By $\mathrm{v}$ and $\mathrm{c}$, we label the LDA eigenvalues $(\varepsilon)$ and eigen- functions $(\psi)$, with $\mathrm{k}$ spanning a $12 \times 12$ mesh in the twodimensional Brillouin zone for the $4 \times 4$ case (increased to $18 \times 18$ for unsupported silicene in the same unit cell). Intraband transitions, responsible for the infrared absorbance (Drude peak) in metallic systems, were not included. It must be stressed that the inclusion of self-energy and excitonic effects has been shown to lead to a large compensation 
between the latter (electron-hole interaction) and the modification of the single-particle excitation energies ${ }^{16}$; also other silicon low-dimensional systems mostly exhibit a blue shift of the absorption peaks when going beyond IP-RPA ${ }^{17}$.

Ultrafast spectroscopy measurements: We excited the sample with a broadband visible pulse, spectrally peaked around $500 \mathrm{~nm}$, obtained from a visible optical parametric amplifier $(\mathrm{OPA})^{18}$. We used as a probe the second harmonic of another OPA. Both OPAs were driven by an amplified Ti:sapphire laser system $(500 \mu \mathrm{J}, 150 \mathrm{fs}, 1 \mathrm{kHz})$. After chirped mirror compression, the duration of the pump pulse was less than $15 \mathrm{fs}$. We measured the probe reflection of the sample with an optical multichannel analyser working at the full repetition rate of the laser source. The acquisition of the pumpperturbed and pump-unperturbed probe spectra allowed us to extract the sample differential reflectivity $\Delta R / R$.
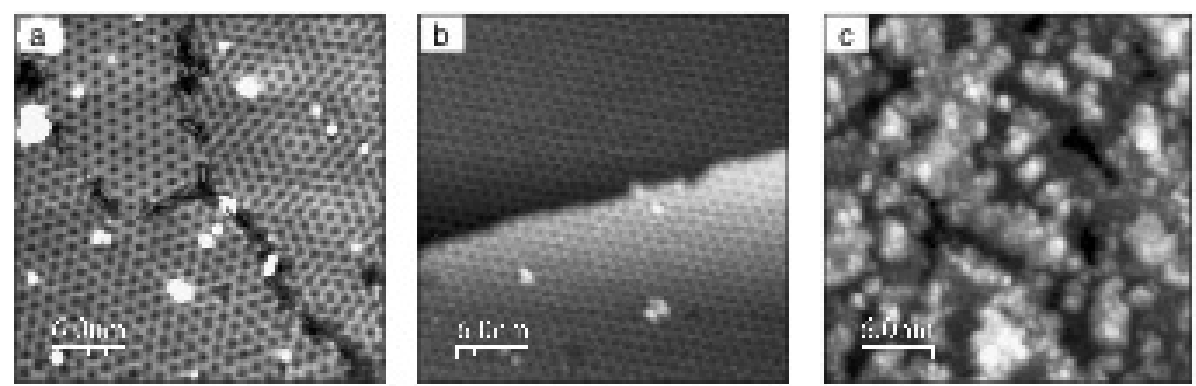

Figure 1. STM image showing the coexisting phases $4 \times 4$ and $\sqrt{13} \times \sqrt{13}$. (b) STM image of the $2 \sqrt{3} \times$ $2 \sqrt{3}$. (c) STM image showing the amorphous Si grown at room temperature. Images are $30 \times 30 \mathrm{~nm}^{2}$ wide and were acquired at sample bias $\mathrm{U}_{\text {bias }}=-1.5 \mathrm{~V},-1 \mathrm{~V}$, and $-1.3 \mathrm{~V}$ and tunneling current $\mathrm{I}=0.35 \mathrm{nA}, 0.64 \mathrm{nA}$, and $0.4 \mathrm{nA}$, respectively.

\section{RESULTS AND DISCUSSION}

Fig. 3compares the typical Raman spectrum of multiphase epitaxial Silicene (where the $4 \times 4$, the $2 \sqrt{3} \times 2 \sqrt{3}$ and $\sqrt{13} \times \sqrt{13}$ superstructures coexists), with those of an isolated $2 \sqrt{3} \times 2 \sqrt{3}$ Silicene phase and of an amorphous silicon (a-Si) control sample. It is worth to notice how the Raman spectrum of the Silicene/ $\mathrm{Ag}(111)$ system is completely different from the one of $1 \mathrm{ML}$ equivalent a-Si, reported as a continuous curve in Fig. 3. This discrepancy can be easily understood if we take into account that silicene possess a crystalline order responsible for the narrow Raman peak (516 $\mathrm{cm}^{-1}$ and $522 \mathrm{~cm}^{-1}$ for the mixed phase and the isolated $2 \sqrt{3} \times 2 \sqrt{3}$ phase respectively), whereas the a-Si bears only short-range order resulting in the typical broad Raman feature peaked at $480 \mathrm{~cm}^{-1}$.

As reported elsewhere ${ }^{5}$, the spectrum in Fig. 3reflects the presence of differently oriented Silicene superstructures having slightly different atomic arrangements. The spectrum shows a sharp and intense $E_{2 \mathrm{~g}}$ mode in the $515-522 \mathrm{~cm}^{-1}$ spectral range plus additional Raman-active and breathing-like $A_{1 g}$ modes having different Raman intensities, accordingly with the buckling distribution of each silicene superstructure. Each Ag-supported silicene phase causes a perturbation of the $D_{3 d}$ point group symmetry of the FS silicene that activates phase specific $A_{1 g}$ modes and bears a phase dependent Raman behaviour as a function of the excitation wavelength ${ }^{5}$. Even tiny modifications on the bond length and bond angle distributions cause remarkable differences in the lattice response following the interaction with an external electric field. 

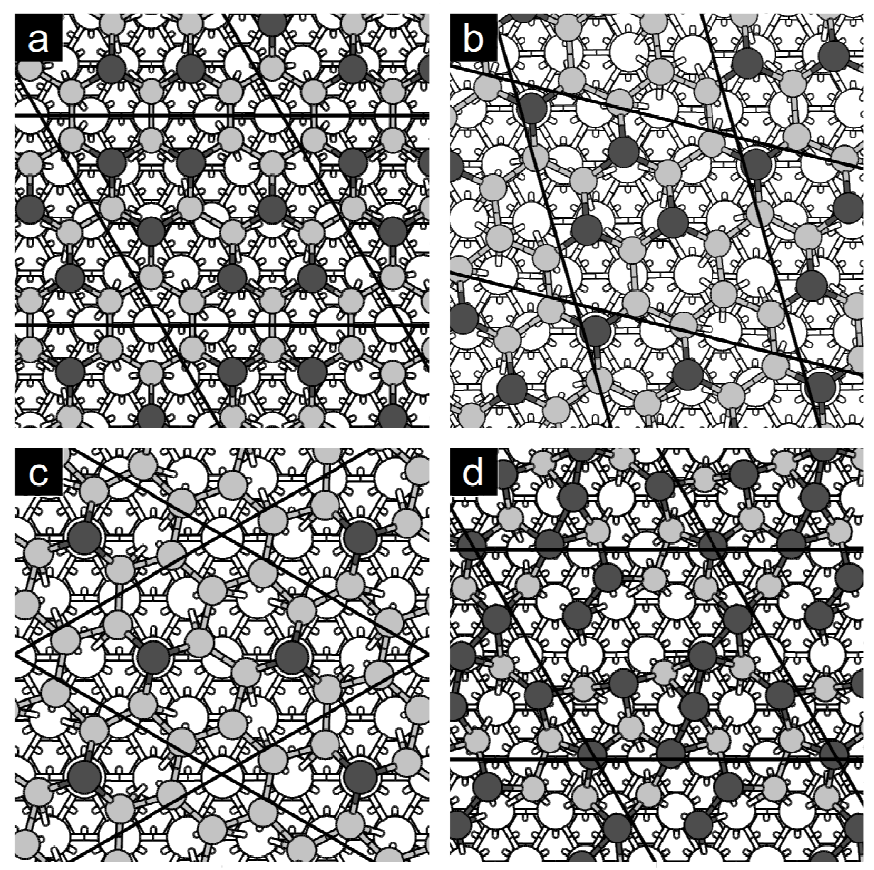

Figure 2. Top view of structural models for the $4 \times 4, \sqrt{13} \times \sqrt{13}$, and $2 \sqrt{3} \times 2 \sqrt{3}$ silicene/ $\operatorname{Ag}(111)$ structures are depicted in panels (a), (b), and (c). Panel (d) shows a structural model for a-Si/Ag(111). Smaller light grey and larger dark grey spheres stand for planar and buckled Si atoms, respectively. The black lines mark the surface unit cells.

Recently, D. Sololenko et al. demonstrated by means of in-situ Raman spectroscopy that the Raman spectrum of epitaxial Silicene is dominated by $\mathrm{A}_{1 \mathrm{~g}}$ modes in the $100-200 \mathrm{~cm}^{-1}$ (a spectral range not accessible in our experiments) that are absent for diamond-like Silicon ${ }^{19}$. The polarized Raman experiment performed by Sololenko and co-workers demonstrated the honeycomb structure of the epitaxial Silicene lattice, thus evidencing how epitaxial Silicene on $\mathrm{Ag}(111)$ represents a new allotropic form of Silicon. This honeycomb structure have been further validate recently by grazing-incidence $\mathrm{x}$-ray diffraction measurements performed for the $(4 \times 4)$ phase by Curcella et al. ${ }^{20}$.

In the framework of optical investigations, the dielectric response of Silicene has been theoretically studied for the unsupported FS phase. Intriguingly, the presence of the buckling, which is responsible for the $\mathrm{sp}^{2}$-sp $\mathrm{sp}^{3}$ character of FSsilicene, does not break the 2D honeycomb lattice point-group symmetry. As a consequence, in analogy with graphene, FS-silicene shows an absorbance proportional to the fine-structure constant in the zero frequency limit $(\omega \rightarrow 0)$ limit. On the other hand, the absorbance of FS-silicene in the UV-Vis spectral range differs significantly from that of graphene because of the different band structures. In particular, the presence of Van Hove singularities in the joint density of state of FS-silicene determines a first absorption peak at $1.6 \mathrm{eV}$. This feature is hugely redshifted with respect to graphene because of the deviation from linearity of FS-silicene $\pi$ - $\pi^{*}$ bands along the $\Gamma M$ direction in the $\mathrm{FBZ}^{14}$.

Concerning epitaxial silicene, the optical properties are completely unexplored in the literature. As aforementioned, epitaxial silicene superstructures present a distorted honeycomb lattice because of the interaction with the substrate and the non-uniform buckling distribution in the unit cell. The role of the $\operatorname{Ag}(111)$ support has been deeply studied in the literature and it has been demonstrated how strong $\mathrm{Si}_{3} \mathrm{P}_{\mathrm{z}}-\mathrm{Ag} 5 \mathrm{~s}$ hybridization effect completely suppress $\pi-\pi^{*}$ bands with the consequent loss of the Dirac cones in the silicene layer ${ }^{6}$. It is thus interesting to elucidate how the support-related loss of symmetry together with the concomitant strong $\mathrm{Si}-\mathrm{Ag}$ interaction reflect in the dielectric response of the $2 \mathrm{D}$ silicene/silver interface. 


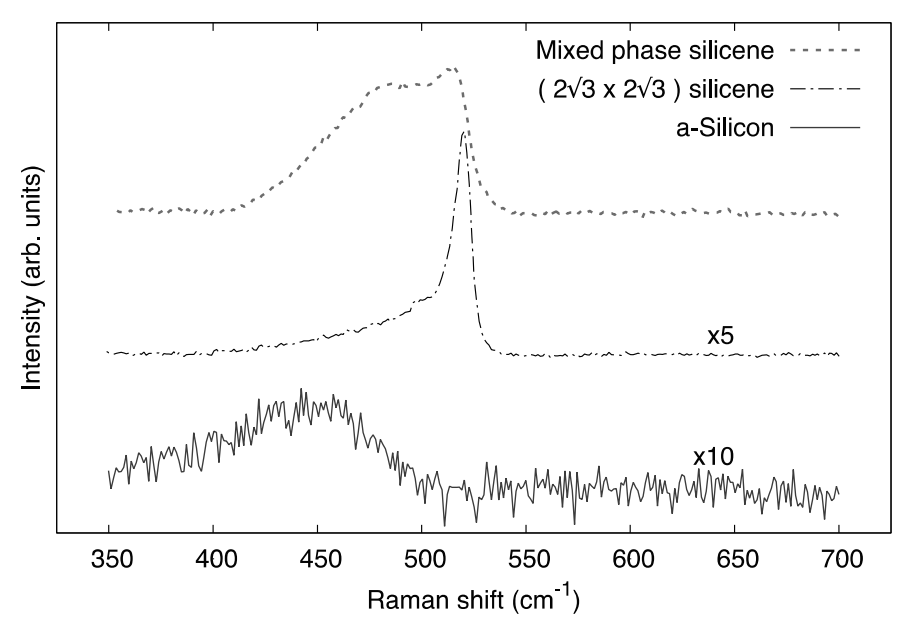

Figure 3. Raman spectra of the mixed phase and $2 \sqrt{3} \times 2 \sqrt{3}$ silicene/ $\operatorname{Ag}(111)$ samples (dotted and dashdotted curve, respectively) compared to a-Si one (solid curve). The strong structure at about $520 \mathrm{~cm}^{-1}$ corresponds to the Raman-active optical modes (stretching modes) of silicene.

Figure 4 shows the calculated absorption spectra of both supported and unsupported $4 \times 4$ and a-Si. It must be stressed that the absence of many-body effects in the modelling, mostly reflects in a blue shift of the absorption peaks, as showed for instance by S. Cahangirov et al. concerning silicon low dimensional systems ${ }^{17}$.

For the unsupported $4 \times 4$ case (dotted curve in Figure 4, an emerging evidence is that the van Hove feature at $1.6 \mathrm{eV}$ at the $M$ point of the FBZ is still present, indicating that the singularity in the band structure at this energy is preserved despite the lower symmetry of the honeycomb lattice. Conversely, the sharp interband peak at $4 \mathrm{eV}$ characterizing FSsilicene absorbance broadens in the $2-6 \mathrm{eV}$ spectral range indicating a modified band structure.

It is worth to notice that, if the symmetry is totally reduced down to the amorphous limit, as for the unsupported aSilicon (dashed line in Figure 4, then all the absorption peaks disappear and the absorbance is almost flat all over the spectral region with a slight maximum at $2 \mathrm{eV}$.

The effect of the silver substrate is not trivial and reflects the special physics of the $2 \mathrm{D}$ Silicene $/ \operatorname{Ag}(111)$ interface. The dash-dotted curve in Figure 4reports the imaginary part of the dielectric function of the bare silver, which makes evidence of a negligible contribution in the visible spectral range and a significant absorbance above $4 \mathrm{eV}$. Interestingly, the presence of the substrate does not trivially recast as a modified background with respect of the unsupported case, as one can expect from the absorbance of the bare $\operatorname{Ag}(111)$. The continuous curve in Figure 4 is the absorbance of $4 \times 4$ Silicene on $\operatorname{Ag}(111)$. Beyond an increased background in the Uv spectral region, two main effects are evident, the absence of the van Hove peak at $1.6 \mathrm{eV}$ combined with the sharpening and the redshifting of the main absorption peak. The former fact highlights how the presence of silver hugely impacts the band structure of the adsorbed silicon, and consequently its absorbance: this finding is consistent with a strong hybridization between $\mathrm{Ag}$ and $\mathrm{Si}$ states, as discussed in the recent literature ${ }^{6,721}$. The latter observation makes further evidence that the electronic transitions involved in the optical absorption cannot be trivially attributed to silicon states only, but include strong Ag contributions extending within the substrate. These effects are apparently absent for the a-Si case, whose absorption profile is weakly influenced by the presence of the underlying silver substrate.

In order to elucidate the role of silver in determining the optical response of epitaxial silicene, we developed a method, which enabled us to single out the contribution to the optical properties of the silicene/silver interface according to the localization of the valence and conduction states involved in the transitions, either on Si or Ag atoms. Such analysis is a clear advantage of the independent-particle approximation that would not be as straightforward for more refined treatments, and allows a qualitative understanding of the spectral features. Hence, we obtain for the slab absorption a factorization into pure $\mathrm{Ag}-\mathrm{Ag}, \mathrm{Si}-\mathrm{Si}$ and mixed $\mathrm{Ag}-\mathrm{Si}$ and $\mathrm{Si}-\mathrm{Ag}$ electronic transitions. These are defined by assigning to each of the valence-to-conduction transition in Eq. (2) a weight equal to the amplitude of the Kohn-Sham states on the respective part. Here, such localization is determined by Lowdin population analysis, namely projecting the Bloch orbitals onto atomic states. Since such projection is not complete in practical applications, a remainder contribution is 
also to be expected but was found to be insignificant here. The results for this procedure are presented in Fig.5a for the $(4 \times 4)$ phase.

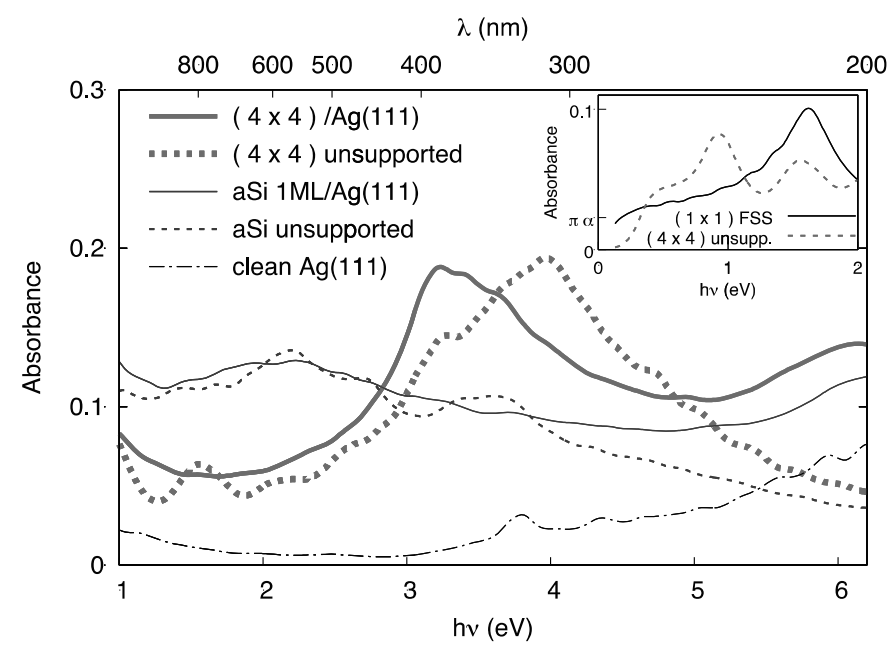

Figure 4. Calculated absorbance of the Ag- supported and unsupported $4 \times 4$ superstructure compared to the supported and unsupported amorphous silicon. The absorbance of the bare Ag slab is also reported. The inset compares the absorbances of $1 \times 1 \mathrm{FSS}$ and unsupported $4 \times 4$ in the infrared range.
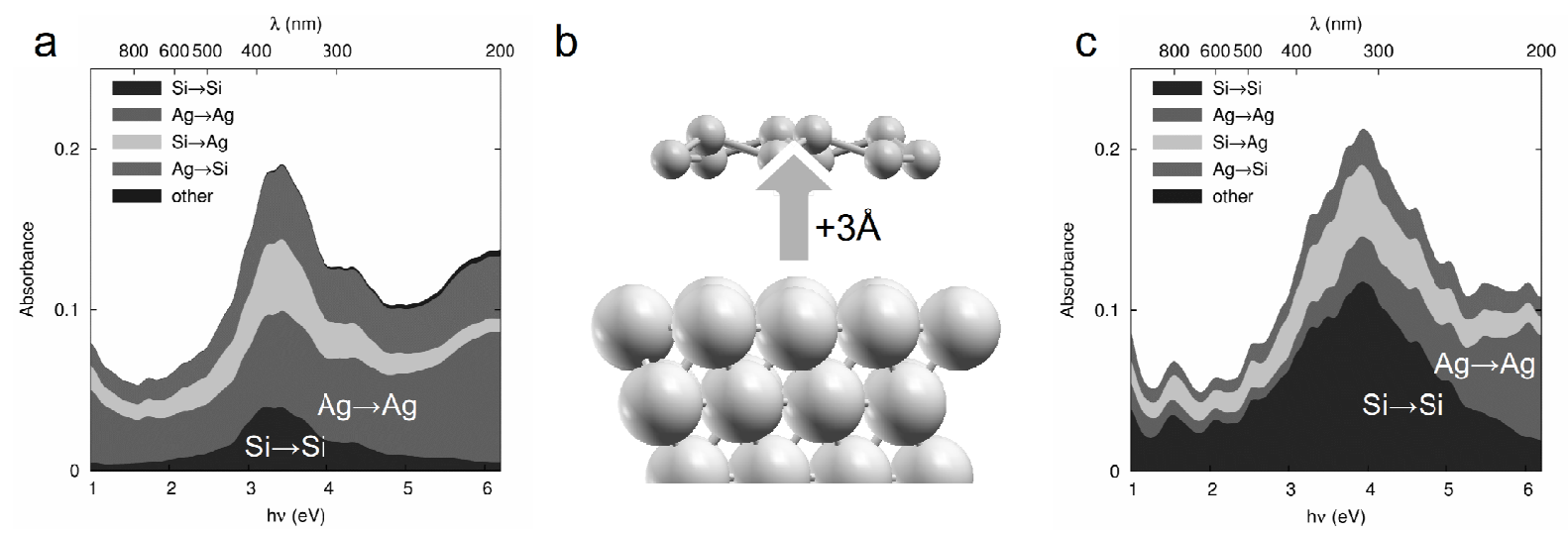

Figure 5 (a) Factorized absorbance into the various valence $\rightarrow$ conduction contributions of the $4 \times 4$ superstructure. (b) Model $4 \times 4$ superstructure where the Si-Ag distance has been artificially increased by $3 \AA$. (c) Factorized absorbance for such model.

An apparently counterintuitive scenario emerges when analysing pure and mixed contributions: the pure silicon states provide just a weak contribution to the total absorption whereas electronic transition involving pure Ag states or mixed $\mathrm{Si}-\mathrm{Ag}$ and $\mathrm{Ag}-\mathrm{Si}$ states are responsible for almost the whole intensity in the spectral range around $3 \mathrm{eV}$ whose absorption spectrum is strictly related to the presence of silicene. On the contrary, its intensity is somehow dominated by silver states, making evidence of a non-trivial delocalization effect influencing silicon-derived wavefunctions at the $\mathrm{Si} / \mathrm{Ag}$ interface. The strong interaction between the adsorbate and the metallic support was already studied by means of electronic spectroscopies, nevertheless the unexpected contributions to the absorption of silver states points out to a nontrivial physics that descends from the reduction of the Si layer thickness down to the 2D limit.

The same analysis has been performed for the other silicene phases, as well as for the model amorphous Silicon ${ }^{8}$. Equivalent results for all silicene phases were obtained, that may not appear surprising given that the interaction with the substrate dominates over the specific arrangement of the allotrope in determining the band structure ${ }^{7}$. However, the factorized absorption of a $1 \mathrm{ML}$ of a-Si on $\mathrm{Ag}(111)$, although the silver substrate does not affect the absorption in such an extent as for silicene (see Fig. 4), also presented the same findings observed as for honeycomb silicene. While the 
absence of modification in the spectrum of supported and unsupported a-Silicon is straightforward, the huge contribution to the absorption of $\mathrm{Ag}$ wavefunctions is remarkable. In complete analogy with the silicene case, a-Silicon optical absorption is dominated by pure $\mathrm{Ag}$ and mixed $\mathrm{Ag}-\mathrm{Si} / \mathrm{Si}-\mathrm{Ag}$ contributions, making evidence that the delocalization of $\mathrm{Si}$ wavefunctions in the silver substrate is a dimensional effect strictly related to the two-dimensional nature of the silicon adsorbate, no matter of its atomic configuration. Such effect may be influenced and controlled by tuning the silicenesubstrate interaction, which is an active field of research for 2D materials. As a proof-of-concept analysis, we artificially modified the $(4 \times 4)$ phase by lifting it by $3 \AA$ (with all other coordinates unchanged, see Fig. $5 \mathrm{~b}$ ). The factorized absorption for this test case is presented in Fig. $5 \mathrm{c}$ and clearly presents a strong enhancement in the relative Si-Si contribution at the $4 \mathrm{eV}$ Si-derived peak, whereas mixed contributions are now significantly reduced in agreement with the expected reduction of hybrid character for silicene/silver states.

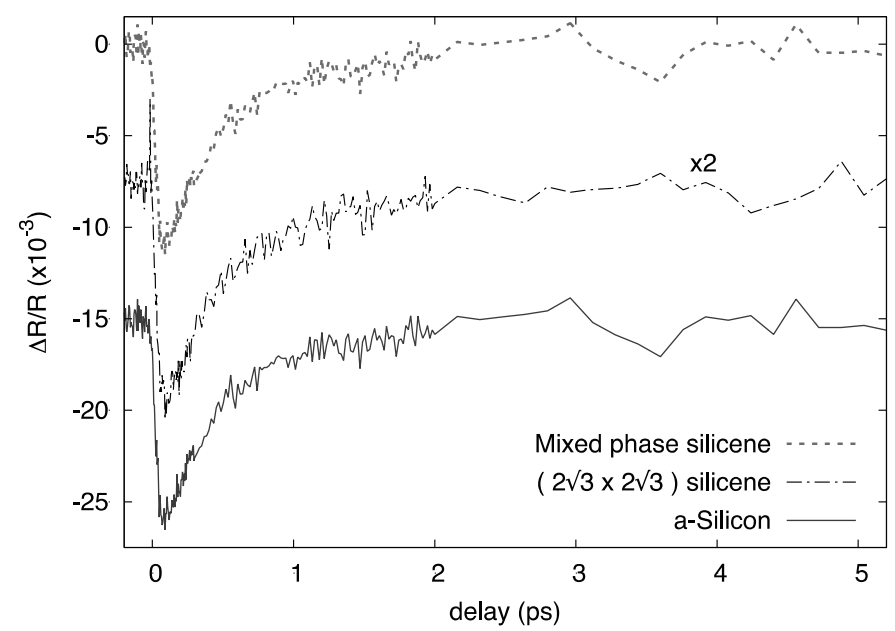

Figure 6 Transient-reflectance spectra as a function of pump-probe delay for the probe wavelength of $340 \mathrm{~nm}$ in mixed-phase silicene/ $\operatorname{Ag}(111)$ (dotted curve), $2 \sqrt{3} \times 2 \sqrt{3}$ silicene/ $\operatorname{Ag}(111)$ (dash-dotted curve), and a$\mathrm{Si} / \operatorname{Ag}(111)$ (solid curve).

The transient reflectivity dynamics $(\Delta R / R)$ of the mixed-phase silicene/Ag(111) (dotted curve), $2 \sqrt{3} \times 2 \sqrt{3}$ silicene/ $\mathrm{Ag}(111)$ (dashdotted curve), and a-Si/ $\mathrm{Ag}(111)$ (continuous curve) are shown in Fig. 6 . The probe wavelength is $340 \mathrm{~nm}$. The temporal behavior at this wavelength is characterized by at ultrafast decay at 2 ps after photoexcitation. Diamond like and amorphous silicon show the typical decay of tens to hundreds of picoseconds due to exciton recombination in indirect and direct band-gap semiconductors, while a fast dynamics of tens of fs, due to electronelectron scattering, is characteristic of metals. The dynamic of silicene on silver is peculiar and can be related both to hybrid silicene/silver behavior and to amorphous silicon behavior. A metallic behavior in silicene/Ag is evident because of the dynamics characterized by a time constant of hundreds of femtoseconds, three orders of magnitude faster than in conventional semiconductors. Although, the dynamic is slower with respect to pure metallic systems. This highlights a behavior characteristic of a hybrid $2 \mathrm{D} \mathrm{Si} / \mathrm{Ag}$ interface, with electron-electron coupling and electron-phonon coupling with distinct time constants, and with the absence of exciton recombination.

\section{CONCLUSION}

In this work we study the optical properties of epitaxial silicene on silver. We demonstrated that the strong hybridization at the Silicon/Silver interface makes trivial any structural difference among differently oriented silicene phases. The contribution of Silver states in the optical absorption of the epitaxial silicene dominates with respect of transitions that involve only Silicon states. Transient-Reflectance spectroscopy allowed us to identify the strong hybridization by observing a metallic-like ultrafast response of epitaxial silicene. 


\section{REFERENCES}

[1] Cahangirov, S., Topsakal, M., Aktürk, E., Şahin, H.., Ciraci, S., "Two- and One-Dimensional Honeycomb Structures of Silicon and Germanium," Phys. Rev. Lett. 102(23) (2009).

[2] Ezawa, M., "Valley-Polarized Metals and Quantum Anomalous Hall Effect in Silicene," Phys. Rev. Lett. 109(5) (2012).

[3] Vogt, P., De Padova, P., Quaresima, C., Avila, J., Frantzeskakis, E., Asensio, M. C., Resta, A., Ealet, B.., Le Lay, G., "Silicene: Compelling Experimental Evidence for Graphenelike Two-Dimensional Silicon," Phys. Rev. Lett. 108(15) (2012).

[4] Chiappe, D., Grazianetti, C., Tallarida, G., Fanciulli, M.., Molle, A., "Local Electronic Properties of Corrugated Silicene Phases,” Adv. Mater. 24(37), 5088-5093 (2012).

[5] Cinquanta, E., Scalise, E., Chiappe, D., Grazianetti, C., van den Broek, B., Houssa, M., Fanciulli, M.., Molle, A., "Getting through the Nature of Silicene: An $\mathrm{sp}^{2}-\mathrm{sp}^{3}$ Two-Dimensional Silicon Nanosheet," J. Phys. Chem. C 117(32), 16719-16724 (2013).

[6] Tsoutsou, D., Xenogiannopoulou, E., Golias, E., Tsipas, P.., Dimoulas, A., "Evidence for hybrid surface metallic band in $(4 \times 4)$ silicene on Ag(111)," Appl. Phys. Lett. 103(23), 231604 (2013).

[7] Sheverdyaeva, P. M., Mahatha, S. K., Moras, P., Petaccia, L., Fratesi, G., Onida, G.., Carbone, C., "Electronic States of Silicene Allotropes on $\operatorname{Ag}(111)$," ACS Nano 11(1), 975-982 (2017).

[8] Cinquanta, E., Fratesi, G., dal Conte, S., Grazianetti, C., Scotognella, F., Stagira, S., Vozzi, C., Onida, G.., Molle, A., "Optical response and ultrafast carrier dynamics of the silicene-silver interface," Phys. Rev. B 92(16), 165427 (2015).

[9] Tao, L., Cinquanta, E., Chiappe, D., Grazianetti, C., Fanciulli, M., Dubey, M., Molle, A.., Akinwande, D., "Silicene field-effect transistors operating at room temperature," Nat. Nanotechnol. 10(3), 227-231 (2015).

[10] Santos, I., Cazzaniga, M., Onida, G.., Colombo, L., "Atomistic study of the structural and electronic properties of a-Si:H/c-Si interfaces," J. Phys. Condens. Matter 26(9), 095001 (2014).

[11] Giannozzi, P., Baroni, S., Bonini, N., Calandra, M., Car, R., Cavazzoni, C., Ceresoli, D., Chiarotti, G. L., Cococcioni, M., et al., "QUANTUM ESPRESSO: a modular and open-source software project for quantum simulations of materials," J. Phys. Condens. Matter 21(39), 395502 (2009).

[12] Perdew, J. P.., Zunger, A., "Self-interaction correction to density-functional approximations for many-electron systems," Phys. Rev. B 23(10), 5048-5079 (1981).

[13] Marini, A., Hogan, C., Grüning, M.., Varsano, D., "yambo: An ab initio tool for excited state calculations," Comput. Phys. Commun. 180(8), 1392-1403 (2009).

[14] Bechstedt, F., Matthes, L., Gori, P.., Pulci, O., "Infrared absorbance of silicene and germanene," Appl. Phys. Lett. 100(26), 261906 (2012).

[15] Onida, G., Reining, L.., Rubio, A., "Electronic excitations: density-functional versus many-body Green'sfunction approaches," Rev. Mod. Phys. 74(2), 601-659 (2002).

[16] Wei, W.., Jacob, T., "Strong many-body effects in silicene-based structures," Phys. Rev. B 88(4) (2013).

[17] Cahangirov, S., Özçelik, V. O., Rubio, A.., Ciraci, S., "Silicite: The layered allotrope of silicon,” Phys. Rev. B 90(8) (2014).

[18] Beutler, M., Ghotbi, M., Noack, F., Brida, D., Manzoni, C.., Cerullo, G., "Generation of high-energy sub-20 fs pulses tunable in the 250-310 nm region by frequency doubling of a high-power noncollinear optical parametric amplifier," Opt. Lett. 34(6), 710 (2009).

[19] Solonenko, D., Gordan, O. D., Lay, G. L., Şahin, H., Cahangirov, S., Zahn, D. R. T.., Vogt, P., “2D vibrational properties of epitaxial silicene on $\operatorname{Ag}(111)$," 2D Mater. 4(1), 015008 (2016).

[20] Curcella, A., Bernard, R., Borensztein, Y., Resta, A., Lazzeri, M.., Prévot, G., "Determining the atomic structure of the $(4 \times 4)$ silicene layer on $\operatorname{Ag}(111)$ by combined grazing-incidence $x$-ray diffraction measurements and first-principles calculations," Phys. Rev. B 94(16) (2016).

[21] Cahangirov, S., Audiffred, M., Tang, P., Iacomino, A., Duan, W., Merino, G.., Rubio, A., "Electronic structure of silicene on $\operatorname{Ag}(111)$ : Strong hybridization effects," Phys. Rev. B 88(3) (2013). 